Article

\title{
Prevalence and Comorbidity of Atopic Dermatitis in Children: A Large-Scale Population Study Based on Real-World Data
}

\author{
Yolanda Gilaberte ${ }^{1}$, Juan Blas Pérez-Gilaberte ${ }^{2}{ }^{(}$, Beatriz Poblador-Plou ${ }^{3}\left({ }^{\circ}\right.$, \\ Kevin Bliek-Bueno ${ }^{4}$, Antonio Gimeno-Miguel ${ }^{3, *,+} \mathbb{D}$ and Alexandra Prados-Torres ${ }^{3,+}$ (D) \\ 1 Department of Dermatology, Miguel Servet University Hospital, IIS Aragón, 50009 Zaragoza, Spain; \\ ygilaberte@gmail.com \\ 2 Department of Dermatology, University of Zaragoza, 50009 Zaragoza, Spain; \\ juanblasperezgilaberte@gmail.com \\ 3 EpiChron Research Group, Aragon Health Sciences Institute (IACS), IIS Aragón, Health Services Research \\ on Chronic Patients Network (REDISSEC), Miguel Servet University Hospital, 50009 Zaragoza, Spain; \\ bpoblador.iacs@aragon.es (B.P.-P.); sprados.iacs@aragon.es (A.P.-T.) \\ 4 Teaching Unit of Preventive Medicine and Public Health, Miguel Servet University Hospital, \\ 50009 Zaragoza, Spain; kbliek@salud.aragon.es \\ * Correspondence: agimenomi.iacs@aragon.es; Tel.: +349-7676-5500 (ext. 5375) \\ + These authors have equal contributed to this work.
}

Received: 28 April 2020; Accepted: 25 May 2020; Published: 28 May 2020

\begin{abstract}
This study aimed at exploring atopic dermatitis (AD) prevalence in children and exhaustively analyzing their comorbidity. We conducted a descriptive analysis of their socio-demographic and comorbidity characteristics in the EpiChron Cohort (Aragón, Spain). Adjusted odds ratios (OR) were calculated for each comorbidity using logistic regression models. In total, 33,591 children had a diagnosis of $\mathrm{AD}$, resulting in an overall prevalence of $15.5 \%$. $\mathrm{AD}$ prevalence was higher in girls compared to boys, in 3-9-year-olds compared to children of other ages, and in Spanish children compared to those of other nationalities. Multimorbidity was present in $43 \%$ of children, with the most frequent chronic comorbidities being asthma (13.1\%), psychosocial disorders (7.9\%), and visual impairment $(7.8 \%)$. Many diseases were, regardless of their prevalence, statistically associated with AD. The strongest associations (odds ratio (OR) (95\% confidence interval (CI))) were found in asthma (2.10 (2.02-2.17)), allergic rhinitis (2.00 (1.91-2.10)), and irritable bowel syndrome (1.90 (1.56-2.31)). A better understanding of the array of comorbidities associated with AD in children might help improve their clinical management. Future longitudinal studies are encouraged to shed light on the potential underlying pathophysiological mechanisms involved in the identified associations.
\end{abstract}

Keywords: atopic dermatitis; children; comorbidity; multimorbidity; prevalence; Spain

\section{Introduction}

Atopic dermatitis $(\mathrm{AD})$ is a chronic inflammatory skin disease of multifactorial etiology characterized by age-specific skin lesions, xerosis, and highly pruritic outbreaks. Usually starting in early childhood, AD significantly reduces patients' quality of life [1,2] and increases healthcare resource use [3]. According to the estimates of the International Study of Asthma and Allergies in Childhood (ISAAC) [4], AD globally affects $15 \%-20 \%$ of children and $1 \%-3 \%$ of adults [5]. These prevalence figures are growing worldwide and show clear geographical variances; $\mathrm{AD}$ is more frequent in industrialized and high-income countries than in agriculture-based ones with low incomes $[6,7]$. Only one study to date exhaustively analyzed AD prevalence during childhood in Spain; it was 
conducted in 2008 using a population-based survey and concluded that AD affects around $30 \%$ of 6-7-year-olds [8].

Patients with $\mathrm{AD}$ are more susceptible to the development of allergic diseases and frequently suffer from multimorbidity (i.e., coexistence of more than one chronic condition). Multiple studies link AD during infancy to the future appearance of asthma and allergic rhinitis, with $80 \%$ of patients eventually developing either of them or both [9]. Food allergies occur in $35 \%$ of children with AD [10], and cutaneous infections and keratoconjunctivitis are more frequent than in the general population [11-13]. Psychiatric and psychological disorders, especially attention deficit hyperactivity disorder (ADHD), depression, or anxiety, are also more frequent in AD patients, possibly due to pruritus-induced lack of sleep, psychological stress, and high levels of proinflammatory cytokines [9]. Other comorbidities of varying nature such as cardiovascular diseases, type 2 diabetes, obesity, infections that go beyond cutaneous over-infection, malignancies (e.g., lymphomas), or autoimmune disorders (e.g., alopecia areata, lupus erythematosus, and inflammatory bowel disease) are also somehow linked to $\mathrm{AD}$ [12,14-16]. To the best of our knowledge, there are no large-scale population-based publications specifically analyzing the global comorbidity of AD during childhood.

Improving our knowledge on the comorbidity profile of children with AD is necessary for their optimal clinical management, which should also consider the concurrence of different comorbidities, and which could shed some light on the underlying pathophysiological mechanisms connecting AD to other diseases. The characterization of the comorbidity profile of this population group should focus not only on the identification of the most common diseases accompanying $\mathrm{AD}$, but also on those that are systematically associated to $\mathrm{AD}$ regardless of their prevalence. The results obtained in this regard could help identify which comorbidities deserve special attention for the development of holistic, person-centered clinical strategies for $\mathrm{AD}$ patients.

The objective of this large-scale, population-based study is to determine the prevalence of AD in children under 18 years of age in a Mediterranean region of Spain, to exhaustively describe their most prevalent comorbidities, and to analyze which diseases display an increased risk of appearance when $\mathrm{AD}$ is present.

\section{Experimental Section}

\subsection{Study Design and Population}

We conducted a retrospective observational study in the EpiChron Cohort, which links socio-demographic and clinical data from all the users of the public health system of the Spanish region of Aragón [17]. This cohort is based on the information registered in the electronic health records (EHRs) and clinical-administrative databases of approximately $98 \%$ of the inhabitants of Aragón (reference population: 1.3 million people). Diagnoses are coded using the International Classification of Primary Care, First Edition (ICPC-1) for primary care health records, or the International Classification of Diseases, Ninth Revision, Clinical Modification (ICD-9-CM) for hospital health records. Diagnoses are subsequently grouped in expanded diagnostic clusters (EDCs) through the Johns Hopkins Adjusted Clinical Groups $\left(\mathrm{ACG}^{\circledR}\right)$ System [18] (version 11.0, The Johns Hopkins University, Baltimore, MD, US). A description of the cohort profile that served as the basis of more than 20 clinical-epidemiological studies on chronic diseases, as well as of the information sources and data curation procedures used, was published elsewhere [17].

For this study, we selected the individuals of the cohort aged 17 and under on 31 December 2015 $(n=216,291)$. Patients with AD were then identified through the appropriate diagnostic codes recorded in their primary (i.e., ICPC-1 code "S87") and/or hospital (i.e., ICD-9-CM code "691', excepting “691.0") EHRs between 1 January 2015 and 31 December 2015. In Spain, AD is diagnosed clinically by dermatologists or pediatricians based on medical history, the morphology and distribution of skin lesions, and associated clinical signs [19]. Only patients living in Aragón and registered as users of the 
public health system during the year prior to the start of the study and during the complete one-year follow-up period were included.

The Clinical Research Ethics Committee of Aragón (CEICA) approved this study (Research protocol PI18/082) and waived the requirement to obtain the informed consent from patients given the epidemiological nature of the project, which used anonymized data.

\subsection{Study Variables}

For each subject, we analyzed the following variables: sex, age as of 31 December 2015, nationality, type of residence according to the type of administrative health area (urban, if $80 \%$ or more of the population of the area is concentrated in one of the municipalities, or rural for the rest), deprivation index of the area according to 26 socio-economic indicators ordered from least (Q1) to most (Q4) deprived areas [20], and all chronic disease diagnoses, as well as those of specific, acute conditions considered clinically relevant in $\mathrm{AD}$ (i.e., allergic rhinitis, conjunctivitis/keratitis, acne, otitis media/externa, pharyngitis and tonsillitis, epistaxis, and acute respiratory tract infection) with at least five cases in the population with AD. Medical conditions were considered chronic if included in the list of 114 EDCs defined by Salisbury et al. as those conditions that normally last six months or more, including past conditions that require ongoing disease or risk management, important conditions with a significant risk of recurrence, or past conditions that have continuing implications for patient management [21]. Multimorbidity was defined as the co-occurrence of two or more conditions from said list.

\subsection{Statistical Analysis}

We performed a descriptive analysis of the frequency and prevalence (\%) of $\mathrm{AD}$ in children based on sex, age (i.e., 0-2-, 3-9-, 10-14-, and 15-17-year-olds), nationality, and residence area. We used logistic regression models to calculate the likelihood of presenting AD according to each of the categories of the aforementioned variables in the form of crude and age- and sex-adjusted odds ratios (ORs). Adjusted ORs were compared setting statistical significance at $p<0.05$. We then analyzed the socio-demographic characteristics and comorbidity profile of children affected by AD. Results were calculated as means and/or frequencies with their corresponding standard deviations and/or 95\% confidence intervals (CI).

For the analysis of AD comorbidity, we described the frequency and prevalence of chronic diseases and of the acute conditions of interest. For the identification of those comorbidities systematically associated with $\mathrm{AD}$, we used logistic regression models to calculate the occurrence risk of each comorbidity (dependent variable) based on the presence or absence of $\mathrm{AD}$ (independent variable). We obtained crude and adjusted ORs for sex, age, deprivation index, and rurality. We compared adjusted ORs using the Bonferroni correction method for multiple comparisons to control familywise error rates (for a total of 82 comparisons of diseases with at least five cases), setting statistical significance at $p<0.00061$. Statistical analyses were conducted using Stata software (Version 12.0, StataCorp LLC, College Station, TX, US).

\section{Results}

\subsection{Prevalence and Socio-Demographics of Atopic Dermatitis in Children}

A total of 33,591 children under 18 years of age belonging to the cohort showed an AD diagnosis during the study period, which resulted in an overall $\mathrm{AD}$ prevalence of $15.5 \%$ (Table 1 ). This prevalence was slightly higher in girls compared to boys (15.8\% vs. $15.3 \%$; OR $1.04,95 \%$ CI $1.01-1.06)$, and in 3-9- (20.8\%; OR 1.98, 95\% CI 1.91-2.06) and 10-14-year-old children (14.0\%; OR 1.26, 95\% CI 1.21-1.31) compared to those aged 0-2 years (Table 2), although 15-17-year-old adolescents showed the lowest prevalence rate $(7.8 \%$; OR $0.64,95 \%$ CI $0.61-0.67)$. Spanish children were more frequently affected by $\mathrm{AD}$ than those of different nationalities (ORs from 0.51 to 0.79 ). Regarding the area of residence, AD prevalence was slightly lower in rural areas (OR 0.95, 95\% CI 0.93-0.97) and in the most deprived ones (OR $0.86,95 \%$ CI $0.84-0.89$ ). The mean age of children with AD was 7.72 years (SD 4.26), and the proportion of boys and girls was similar (Table 3 ). 
Table 1. Frequency and prevalence (\%) of atopic dermatitis (AD) in children of the EpiChron Cohort aged 0-17 years in 2015 according to sex, age, nationality, area of residence and deprivation index. EU-European Union.

\begin{tabular}{cccc}
\hline & Boys $(\boldsymbol{n}=\mathbf{1 1 0 , 9 3 4 )}$ & Girls $(\boldsymbol{n = 1 0 5 , 3 5 7 )}$ & Total $(\boldsymbol{n = 2 1 6 , 2 9 1 )}$ \\
\hline AD Prevalence & $\boldsymbol{n} \mathbf{( \% )}$ & $\boldsymbol{n} \mathbf{( \% )}$ & $\boldsymbol{n} \mathbf{( \% )}$ \\
\hline Sex & $16,968(15.3)$ & $16,623(15.8)$ & $33,591(15.5)$ \\
Age (years) & & & \\
$0-2$ & $2110(12.5)$ & $1704(10.7)$ & $3814(11.7)$ \\
$3-9$ & $9320(20.5)$ & $9230(21.1)$ & $18,550(20.8)$ \\
$10-14$ & $4225(13.7)$ & $4358(14.8)$ & $8583(14.2)$ \\
$15-17$ & $1313(7.42)$ & $1331(8.19)$ & $2644(7.79)$ \\
Nationality & & & \\
Spain & $15,184(16.1)$ & $14,863(16.6)$ & $30,047(16.3)$ \\
Eastern Europe & $685(10.0)$ & $714(10.8)$ & $1399(10.4)$ \\
Asia & $150(13.0)$ & $145(13.6)$ & $295(13.3)$ \\
North Africa & $459(11.6)$ & $423(11.4)$ & $882(11.5)$ \\
Sub-Saharan Africa & $201(13.3)$ & $197(14.9)$ & $398(14.1)$ \\
Latin America & $232(10.2)$ & $223(9.98)$ & $455(10.1)$ \\
EU and North America & $52(8.54)$ & $54(8.72)$ & $106(8.63)$ \\
Area of residence ${ }^{1}$ & & & \\
Urban & $10,297(15.5)$ & $10,321(16.2)$ & $20,496(15.7)$ \\
Rural & $6671(15.0)$ & $6302(15.2)$ & $13,095(15.2)$ \\
Deprivation index ${ }^{2}$ & & & \\
$\mathrm{Q}_{1}$ & $5259(16.4)$ & $5026(16.4)$ & $10,285(16.4)$ \\
$\mathrm{Q}_{2}$ & $4085(15.3)$ & $4112(16.0)$ & $8197(15.7)$ \\
$\mathrm{Q}_{3}$ & $3356(15.4)$ & $3316(16.2)$ & $6672(15.8)$ \\
$\mathrm{Q}_{4}$ & $4268(14.0)$ & $4169(14.6)$ & $8437(14.3)$ \\
\hline
\end{tabular}

${ }^{1}$ Based on the type of administrative health area (urban, if $80 \%$ or more of the population of the area is concentrated in one of the municipalities, or rural for the rest); ${ }^{2}$ calculated at aggregated level per administrative health area according to 26 socio-economic indicators and divided into quartiles from less (Q1) to more (Q4) deprived.

Table 2. Likelihood of presenting atopic dermatitis based on sex, age, nationality, area of residence (urban/rural), and deprivation index of the area, calculated using logistic regression models.

\begin{tabular}{|c|c|c|c|c|}
\hline Variable & Crude OR ${ }^{1}$ & $p$-Value $^{2}$ & Adjusted OR ${ }^{3}$ & $p$-Value \\
\hline \multicolumn{5}{|l|}{ Sex } \\
\hline Boys & Reference & & & \\
\hline Girls & $1.04(1.01-1.06)$ & 0.002 & & \\
\hline \multicolumn{5}{|l|}{ Age (years) } \\
\hline $0-2$ & Reference & & & \\
\hline $3-9$ & $1.98(1.91-2.06)$ & $<0.001$ & & \\
\hline $10-14$ & $1.26(1.21-1.31)$ & $<0.001$ & & \\
\hline 15-17 & $0.64(0.61-0.67)$ & $<0.001$ & & \\
\hline \multicolumn{5}{|l|}{ Nationality } \\
\hline Spain & Reference & & Reference & \\
\hline Sub-Saharan Africa & $0.84(0.76-0.94)$ & 0.001 & $0.79(0.71-0.88)$ & $<0.001$ \\
\hline Asia & $0.78(0.69-0.89)$ & $<0.001$ & $0.76(0.67-0.86)$ & $<0.001$ \\
\hline Eastern Europe & $0.59(0.56-0.63)$ & $<0.001$ & $0.59(0.56-0.62)$ & $<0.001$ \\
\hline Latin America & $0.57(0.52-0.63)$ & $<0.001$ & $0.61(0.55-0.67)$ & $<0.001$ \\
\hline North Africa & $0.67(0.62-0.72)$ & $<0.001$ & $0.63(0.58-0.67)$ & $<0.001$ \\
\hline EU and North America & $0.48(0.40-0.59)$ & $<0.001$ & $0.51(0.41-0.62)$ & $<0.001$ \\
\hline \multicolumn{5}{|l|}{ Area of residence } \\
\hline Urban & Reference & & Reference & \\
\hline Rural & $0.95(0.93-0.97)$ & $<0.001$ & $0.95(0.93-0.97)$ & $<0.001$ \\
\hline \multicolumn{5}{|l|}{ Deprivation index ${ }^{4}$} \\
\hline $\mathrm{Q}_{1}$ & Reference & & Reference & \\
\hline $\mathrm{Q}_{2}$ & $0.95(0.92-0.98)$ & 0.001 & $0.97(0.94-1.00)$ & 0.081 \\
\hline $\mathrm{Q}_{3}$ & $0.96(0.92-0.99)$ & 0.008 & 0.97 (0.94-1.00) & 0.099 \\
\hline $\mathrm{Q}_{4}$ & $0.85(0.82-0.88)$ & $<0.001$ & $0.86(0.84-0.89)$ & $<0.001$ \\
\hline
\end{tabular}

${ }^{1}$ Odds ratio; ${ }^{2} p$-values less than 0.05 were considered significant and are highlighted in bold; ${ }^{3}$ adjusted odds ratios for age and sex; ${ }^{4}$ calculated at aggregated level per administrative health area according to 26 socio-economic indicators and divided into quartiles from less (Q1) to more (Q4) deprived. 
Table 3. Socio-demographic and clinical characteristics of children of the EpiChron Cohort aged 0-17 years with atopic dermatitis in 2015.

\begin{tabular}{|c|c|c|c|}
\hline Characteristics & Boys & Girls & Total \\
\hline$n(\%)$ & $16,968(50.5)$ & $16,623(49.5)$ & $33,591(100)$ \\
\hline Mean age, years $\left(\mathrm{SD}^{1}\right)$ & $7.61(4.29)$ & $7.83(4.24)$ & $7.72(4.26)$ \\
\hline \multicolumn{4}{|l|}{ Age group, years $(n, \%)$} \\
\hline $0-2$ & $2110(12.4)$ & $1704(10.2)$ & 3814 (11.3) \\
\hline $3-9$ & $9320(54.9)$ & $9230(55.5)$ & $18,550(55.2)$ \\
\hline $10-14$ & $4225(24.9)$ & $4358(26.2)$ & $8583(25.6)$ \\
\hline $15-17$ & $1313(7.74)$ & $1331(8.01)$ & $2644(7.87)$ \\
\hline \multicolumn{4}{|l|}{ Nationality $(n, \%)$} \\
\hline Spain & $15,184(89.5)$ & $14,863(89.4)$ & $30,047(89.5)$ \\
\hline Eastern Europe & $685(4.04)$ & $714(4.30)$ & 1399 (4.17) \\
\hline Asia & $150(0.88)$ & $145(0.87)$ & $295(0.88)$ \\
\hline North Africa & $459(2.71)$ & $423(2.55)$ & $882(2.63)$ \\
\hline Sub-Saharan Africa & $201(1.18)$ & $197(1.19)$ & $398(1.19)$ \\
\hline Latin America & $232(1.37)$ & $223(1.34)$ & $455(1.35)$ \\
\hline EU and North America & $52(0.31)$ & $54(0.32)$ & $106(0.32)$ \\
\hline \multicolumn{4}{|l|}{ Area of residence } \\
\hline $\operatorname{Urban}^{2}(n, \%)$ & $10,297(60.7)$ & $10,321(62.1)$ & $20,618(61.4)$ \\
\hline \multicolumn{4}{|l|}{ Deprivation index ${ }^{3}(n, \%)$} \\
\hline $\mathrm{Q}_{1}$ & $5259(31.0)$ & $5026(30.2)$ & $10,285(30.6)$ \\
\hline$\hat{\mathrm{Q}}_{2}$ & $4085(24.1)$ & $4112(24.7)$ & $8197(24.4)$ \\
\hline$\hat{\mathrm{Q}}_{3}$ & $3356(19.8)$ & $3316(19.9)$ & $6672(19.9)$ \\
\hline $\mathrm{Q}_{4}$ & $4268(25.1)$ & $4169(25.1)$ & $8437(25.1)$ \\
\hline $\begin{array}{l}\text { Number of chronic diseases } \\
\text { (mean, SD) }\end{array}$ & $1.67(0.91)$ & $1.58(0.85)$ & $1.62(0.88)$ \\
\hline Multimorbidity ${ }^{4}$, yes $(n, \%)$ & $7773(45.8)$ & $6794(40.9)$ & $(43.4)$ \\
\hline
\end{tabular}

${ }^{1}$ Standard deviation; ${ }^{2}$ versus rural; ${ }^{3}$ calculated at aggregated level per administrative health area according to 26 socio-economic indicators and divided into quartiles from less $(\mathrm{Q} 1)$ to more $(\mathrm{Q} 4)$ deprived; ${ }^{4}$ defined as the presence of two or more chronic diseases from a list of 114 conditions.

\subsection{Comorbidity of Atopic Dermatitis in Children}

Over $40 \%$ of children with AD showed multimorbidity, with a mean disease burden of 1.62 (SD 0.88) chronic diseases (Table 3). The most frequent chronic comorbidities in children with AD of all ages and for both sexes were asthma (13.1\%), psychosocial disorders $(7.9 \%)$, visual impairment $(7.8 \%)$, congenital anomalies of limbs (5.8\%), and developmental disorders (3.2\%), among others. All these comorbidities were more prevalent in the population with AD (Table 4). Upper respiratory tract infections $(54.4 \%)$, otitis media $(12.8 \%)$, conjunctivitis $(10.1 \%)$, and pharyngitis $(7.9 \%)$ were amongst the most prevalent acute conditions in this atopic cohort. The prevalence of both acute and chronic comorbidities varied based on sex and age group (Table S1, Supplementary Materials), with notable differences such as the earlier and more frequent occurrence of asthma, psycho-physiologic and somatoform disorders in boys than girls.

Regardless of their prevalence and after adjusting by sex, age, rurality, and deprivation index, the conditions that $\mathrm{AD}$ was significantly associated to the most were the following: (OR (95\% CI) ) asthma (2.10 (2.02-2.17)), allergic rhinitis (2.00 (1.91-2.10)), and irritable bowel syndrome (1.90 (1.56-2.31)). A large and diverse number of comorbidities (i.e., cutaneous, neuropsychological, musculoskeletal) were also significantly more prevalent in the atopic population (Table 4). Chronic cutaneous disorders most associated to AD included psoriasis (1.62 (1.30-2.02)) and diseases of hair and hair follicles, including alopecia and seborrheic dermatitis (1.51 (1.38-1.64)). Childhood psychosocial disorders (1.53 (1.46-1.60)), developmental disorders (1.36 (1.27-1.45)), attention deficit disorder (1.37 (1.24-1.52)), and nonorganic sleep disorders (1.72 (1.55-1.90)) were the neuropsychological conditions most associated with AD. Impulse control (1.56 (1.13-2.15)), psycho-physiologic and somatoform disorders (1.47 (1.05-2.07)), and eating disorder (1.43 (1.12-1.83)) also presented high ORs, although not 
significant after applying the Bonferroni correction. Musculoskeletal comorbidities most frequent in AD children included congenital anomalies of limbs, hands, and feet (1.53 (1.45-1.61)), kyphoscoliosis (1.32 (1.22-1.42)), degenerative joint disease (1.42 (1.24-1.64)), and lower back pain (1.38 (1.15-1.65)). Metabolic diseases such as obesity (1.18 (1.07-1.29)) and disorders of lipid metabolism (1.46 (1.32-1.63)) were also significantly more prevalent in the atopic population, whereas cardiac ones such as congenital heart disease (1.25 (1.09-1.43)), cardiac valve disorders (1.57 (1.12-2.20)), and congestive heart failure (1.56 (1.00-2.43)), as well as hematologic disorders like lymphoma and anemia (1.40 (1.14-1.71)), presented high although not significant ORs.

Table 4. Prevalence of chronic comorbidities and of specific acute conditions (in italics) in children aged 0-17 years with atopic dermatitis (AD) in the EpiChron Cohort in $2015(n=33,591)$. Logistic regression models were used to calculate odds ratios (OR) of prevalence for each comorbidity (dependent variable) according to the presence or not of AD (independent variable). HIV-human immunodeficiency virus; AIDS-acquired immune deficiency syndrome.

\begin{tabular}{|c|c|c|c|c|c|}
\hline \multirow{2}{*}{ EDC $^{1}$} & \multirow{2}{*}{ Comorbidity } & Prevalence $^{2}$ & Crude OR $^{3}$ & Adjusted OR $^{5}$ & \multirow[t]{2}{*}{$p$-Value ${ }^{6}$} \\
\hline & & $(n, \%)$ & $\left(95 \% \mathrm{CI}^{4}\right)$ & $(95 \% \mathrm{CI})$ & \\
\hline EAR11 & Acute upper respiratory tract infection & $18,256(54.4)$ & $1.52(1.48-1.55)$ & $1.42(1.39-1.46)$ & 0.0000 \\
\hline ALL04/05 & Asthma & $4384(13.05)$ & $1.94(1.87-2.01)$ & $2.10(2.02-2.17)$ & 0.0000 \\
\hline EAR01 & Otitis media & $4287(12.8)$ & $1.52(1.47-1.58)$ & $1.45(1.40-1.51)$ & 0.0000 \\
\hline EYE07 & Conjunctivitis, keratitis & $3388(10.1)$ & $1.45(1.39-1.51)$ & $1.39(1.33-1.44)$ & 0.0000 \\
\hline EAR09 & Pharyngitis and tonsillitis & $2662(7.92)$ & $1.67(1.60-1.75)$ & $1.74(1.67-1.83)$ & 0.0000 \\
\hline PSY14 & Psychosocial disorders of childhood & $2640(7.86)$ & $1.34(1.28-1.40)$ & $1.53(1.46-1.60)$ & 0.0000 \\
\hline EYE02 & Visual impairment & $2621(7.80)$ & $1.24(1.19-1.30)$ & $1.48(1.42-1.55)$ & 0.0000 \\
\hline ALL03 & Allergic rhinitis & $2386(7.10)$ & $1.50(1.43-1.57)$ & $2.00(1.91-2.10)$ & 0.0000 \\
\hline MUS11 & Congenital anomalies of limbs & $1952(5.81)$ & $1.53(1.46-1.61)$ & $1.53(1.45-1.61)$ & 0.0000 \\
\hline EAR06 & Otitis externa & $1197(3.56)$ & $1.49(1.39-1.59)$ & $1.47(1.37-1.56)$ & 0.0000 \\
\hline NUR19 & Developmental disorder & $1080(3.22)$ & $1.36(1.27-1.45)$ & $1.36(1.27-1.45)$ & 0.0000 \\
\hline SKN04 & Acne & $940(2.80)$ & $0.74(0.70-0.80)$ & $1.18(1.10-1.27)$ & 0.0000 \\
\hline MUS06 & Kyphoscoliosis & $816(2.43)$ & $0.88(0.82-0.95)$ & $1.32(1.22-1.42)$ & 0.0000 \\
\hline SKN13 & Disease of hair and hair follicles & $684(2.04)$ & $1.31(1.21-1.43)$ & $1.51(1.38-1.64)$ & 0.0000 \\
\hline END05 & Other endocrine disorders & $671(2.00)$ & $1.04(0.96-1.13)$ & $1.21(1.12-1.32)$ & 0.0000 \\
\hline EAR08 & Deafness, hearing loss & $652(1.94)$ & $1.41(1.29-1.54)$ & $1.52(1.39-1.66)$ & 0.0000 \\
\hline PSY19 & Sleep disorders, nonorganic origin & $519(1.55)$ & $1.76(1.59-1.94)$ & $1.72(1.55-1.90)$ & 0.0000 \\
\hline PSY05 & Attention deficit disorder & $484(1.44)$ & $1.03(0.94-1.14)$ & $1.37(1.24-1.52)$ & 0.0000 \\
\hline CAR11 & Disorders of lipid metabolism & $422(1.26)$ & $1.21(1.09-1.34)$ & $1.46(1.32-1.63)$ & 0.0000 \\
\hline EAR10 & Epistaxis & $331(0.99)$ & $1.56(1.38-1.76)$ & $1.57(1.39-1.78)$ & 0.0000 \\
\hline MUS03 & Degenerative joint disease & $238(0.71)$ & $1.02(0.89-1.17)$ & $1.42(1.24-1.64)$ & 0.0000 \\
\hline GAS09 & Irritable bowel syndrome & $132(0.39)$ & $1.83(1.51-2.24)$ & $1.90(1.56-2.31)$ & 0.0000 \\
\hline SKN12 & Psoriasis & $102(0.30)$ & $1.24(1.00-1.54)$ & $1.62(1.30-2.02)$ & 0.0000 \\
\hline RES04 & $\mathrm{COPD}^{7}$ & $66(0.20)$ & $1.72(1.30-2.27)$ & $1.76(1.33-2.32)$ & 0.0001 \\
\hline NUT03 & Obesity & $566(1.68)$ & $0.91(0.84-1.00)$ & $1.18(1.07-1.29)$ & 0.0005 \\
\hline MUS14 & Lower back pain & $141(0.42)$ & $0.91(0.76-1.08)$ & $1.38(1.15-1.65)$ & 0.0006 \\
\hline HEM08 & Hematologic disorders, other & $118(0.35)$ & $1.39(1.14-1.71)$ & $1.40(1.14-1.71)$ & 0.0012 \\
\hline CAR04 & Congenital heart disease & $250(0.74)$ & $1.31(1.14-1.50)$ & $1.25(1.09-1.43)$ & 0.0017 \\
\hline END04 & Hypothyroidism & $167(0.50)$ & $1.02(0.86-1.20)$ & $1.28(1.08-1.52)$ & 0.0036 \\
\hline PSY15 & Eating disorder & $79(0.24)$ & $1.13(0.88-1.43)$ & $1.43(1.12-1.83)$ & 0.0041 \\
\hline PSY16 & Impulse control & $47(0.14)$ & $1.22(0.89-1.68)$ & $1.56(1.13-2.15)$ & 0.0066 \\
\hline CAR06 & Cardiac valve disorders & $44(0.13)$ & $1.48(1.06-2.06)$ & $1.57(1.12-2.20)$ & 0.0083 \\
\hline RES11 & Respiratory disorders, other & $118(0.35)$ & $1.37(1.12-1.68)$ & $1.31(1.07-1.61)$ & 0.0087 \\
\hline GSU06 & Chronic cystic disease of the breast & $7(0.02)$ & $1.36(0.59-3.11)$ & $2.61(1.13-6.06)$ & 0.0253 \\
\hline PSY17 & Psycho-physiological and somatoform disorders & $42(0.13)$ & $1.28(0.92-1.80)$ & $1.47(1.05-2.07)$ & 0.0255 \\
\hline GAS02 & Inflammatory bowel disease & $13(0.04)$ & $1.31(0.71-2.40)$ & $1.86(1.01-3.45)$ & 0.0473 \\
\hline CAR05 & Congestive heart failure & $25(0.07)$ & $1.40(0.90-2.18)$ & $1.56(1.00-2.43)$ & 0.0496 \\
\hline CAR09 & Cardiac arrhythmia & $69(0.21)$ & $1.20(0.92-1.55)$ & $1.29(0.99-1.67)$ & 0.0599 \\
\hline GUR10 & Prostatitis & $14(0.04)$ & $1.77(0.97-3.24)$ & $1.77(0.97-3.25)$ & 0.0635 \\
\hline GTC01 & Chromosomal anomalies & $79(0.24)$ & $1.23(0.96-1.57)$ & $1.25(0.98-1.60)$ & 0.0729 \\
\hline MAL03 & High impact malignant neoplasms & $93(0.28)$ & $1.03(0.82-1.28)$ & $1.22(0.98-1.53)$ & 0.0805 \\
\hline EYE13 & Diabetic retinopathy & $16(0.05)$ & $1.67(0.96-2.93)$ & $1.64(0.93-2.87)$ & 0.0849 \\
\hline END06-09 & $\begin{array}{l}\text { Diabetes } \\
\text { Diab }\end{array}$ & $81(0.24)$ & $1.09(0.85-1.38)$ & $1.23(0.97-1.56)$ & 0.0923 \\
\hline GSU08 & Varicose veins of lower extremities & $17(0.05)$ & $1.13(0.67-1.90)$ & $1.55(0.91-2.63)$ & 0.1053 \\
\hline NUR21 & Neurologic disorders, other & $200(0.60)$ & $1.12(0.96-1.31)$ & $1.13(0.97-1.32)$ & 0.1114 \\
\hline GUR03 & Hypospadias, penile anomalies & $106(0.32)$ & $1.23(1.00-1.52)$ & $1.19(0.96-1.47)$ & 0.1116 \\
\hline MAL12 & Malignant neoplasms, colorectal & $1(0.00)$ & $0.21(0.03-1.54)$ & $0.21(0.03-1.55)$ & 0.1258 \\
\hline
\end{tabular}


Table 4. Cont.

\begin{tabular}{|c|c|c|c|c|c|}
\hline \multirow{2}{*}{ EDC $^{1}$} & \multirow{2}{*}{ Comorbidity } & Prevalence $^{2}$ & Crude OR ${ }^{3}$ & Adjusted OR ${ }^{5}$ & \multirow{2}{*}{$p$-Value ${ }^{6}$} \\
\hline & & $(n, \%)$ & $\left(95 \% \mathrm{CI}^{4}\right)$ & $(95 \%$ CI $)$ & \\
\hline END02 & Osteoporosis & $5(0.01)$ & $1.60(0.59-4.34)$ & $2.15(0.78-5.92)$ & 0.1387 \\
\hline HEM01 & Hemolytic anemia & $86(0.26)$ & $1.09(0.87-1.38)$ & $1.19(0.94-1.50)$ & 0.1515 \\
\hline REN04 & Nephritis, nephrosis & $36(0.11)$ & $1.18(0.82-1.69)$ & $1.29(0.90-1.85)$ & 0.1698 \\
\hline CAR14/15 & Hypertension & $37(0.11)$ & $1.01(0.71-1.43)$ & $1.28(0.90-1.82)$ & 0.1770 \\
\hline MAL01 & Malignant neoplasms of skin & $8(0.02)$ & $1.40(0.65-3.05)$ & $1.69(0.77-3.70)$ & 0.1927 \\
\hline NUR08 & Multiple sclerosis & $5(0.01)$ & $1.81(0.66-4.99)$ & $1.95(0.70-5.40)$ & 0.1995 \\
\hline GAS08 & Gastroesophageal reflux & $7(0.02)$ & $0.62(0.29-1.36)$ & $0.60(0.28-1.32)$ & 0.2046 \\
\hline RHU03 & Arthropathy & $7(0.02)$ & $1.81(0.77-4.27)$ & $1.72(0.73-4.04)$ & 0.2157 \\
\hline INF04 & HIV infection, AIDS & $19(0.06)$ & $1.40(0.84-2.31)$ & $1.37(0.83-2.27)$ & 0.2190 \\
\hline HEM03 & Thrombophlebitis & $7(0.02)$ & $0.56(0.26-1.22)$ & $0.62(0.28-1.36)$ & 0.2332 \\
\hline RES06 & Sleep apnea & $12(0.04)$ & $1.48(0.78-2.81)$ & $1.43(0.75-2.71)$ & 0.2731 \\
\hline PSY08 & Personality disorders & $41(0.12)$ & $0.80(0.58-1.12)$ & $1.16(0.84-1.62)$ & 0.3687 \\
\hline GSU11 & Peripheral vascular disease & $7(0.02)$ & $1.12(0.50-2.53)$ & $1.44(0.63-3.28)$ & 0.3872 \\
\hline ALL06 & Disorders of the immune system & $34(0.10)$ & $1.12(0.77-1.62)$ & $1.15(0.79-1.67)$ & 0.4602 \\
\hline CAR03 & Ischemic heart disease (excluding $\mathrm{AMI}^{8}$ ) & $17(0.05)$ & $0.86(0.52-1.44)$ & $0.83(0.50-1.39)$ & 0.4847 \\
\hline REC03 & Chronic ulcer of the skin & $10(0.03)$ & $0.73(0.38-1.42)$ & $0.79(0.41-1.54)$ & 0.4899 \\
\hline NUR07 & Seizure disorder & $222(0.66)$ & $1.01(0.87-1.16)$ & $1.05(0.91-1.21)$ & 0.5276 \\
\hline GTC02 & Inherited metabolic disorders & $5(0.01)$ & $0.80(0.31-2.05)$ & $0.76(0.30-1.95)$ & 0.5685 \\
\hline HEM02 & Iron deficiency anemia & $253(0.75)$ & $0.96(0.84-1.10)$ & $1.04(0.91-1.19)$ & 0.5794 \\
\hline REN05 & Renal disorders, other & $26(0.08)$ & $1.02(0.67-1.56)$ & $1.13(0.74-1.72)$ & 0.5819 \\
\hline PSY07 & Schizophrenia, affective psychosis & $51(0.15)$ & $0.99(0.74-1.34)$ & $1.09(0.81-1.47)$ & 0.5829 \\
\hline PSY02 & Substance use & $35(0.10)$ & $0.71(0.50-1.01)$ & $1.09(0.76-1.56)$ & 0.6293 \\
\hline HEM07 & Hemophilia, coagulation disorder & $6(0.02)$ & $1.21(0.50-2.93)$ & $1.20(0.49-2.91)$ & 0.6909 \\
\hline NUR03 & Peripheral neuropathy, neuritis & $43(0.13)$ & $0.90(0.65-1.25)$ & $0.94(0.68-1.30)$ & 0.7011 \\
\hline EYE06 & Cataract, aphakia & $9(0.03)$ & $0.83(0.41-1.67)$ & $0.87(0.43-1.77)$ & 0.7051 \\
\hline NUR16 & Spinal cord injury/disorders & $11(0.03)$ & $0.92(0.49-1.74)$ & $0.89(0.47-1.68)$ & 0.7091 \\
\hline MAL16 & Acute leukemia & $8(0.02)$ & $0.78(0.37-1.63)$ & $0.88(0.42-1.86)$ & 0.7403 \\
\hline PSY09 & Depression & $44(0.13)$ & $0.67(0.49-0.92)$ & $1.05(0.76-1.44)$ & 0.7751 \\
\hline CAR16 & Cardiovascular disorders, other & $79(0.24)$ & $0.96(0.76-1.22)$ & $1.04(0.81-1.32)$ & 0.7795 \\
\hline GUR09 & Renal calculi & $14(0.04)$ & $1.02(0.57-1.80)$ & $1.08(0.61-1.93)$ & 0.7817 \\
\hline ADM02 & Surgical aftercare & $25(0.07)$ & $0.99(0.64-1.51)$ & $1.06(0.69-1.63)$ & 0.7922 \\
\hline NUR05 & Cerebrovascular disease & $22(0.07)$ & $1.04(0.66-1.64)$ & $1.04(0.66-1.64)$ & 0.8678 \\
\hline GUR01 & Vesicoureteral reflux & $6(0.02)$ & $0.99(0.41-2.36)$ & $1.07(0.45-2.58)$ & 0.8768 \\
\hline INF01 & Tuberculosis infection & $79(0.24)$ & $0.88(0.69-1.11)$ & $1.01(0.80-1.29)$ & 0.9155 \\
\hline PSY01 & Anxiety, neuroses & $72(0.21)$ & $0.69(0.54-0.89)$ & $1.00(0.78-1.28)$ & 0.9718 \\
\hline
\end{tabular}

${ }^{1}$ Expanded diagnostic clusters; ${ }^{2}$ comorbidities appear in descending order of the $p$-value of the comparison; only diseases with a frequency of five or more cases are represented; ${ }^{3}$ confidence interval; ${ }^{4}$ unadjusted odds ratios; ${ }^{5}$ odds ratios adjusted by sex, age, deprivation index and area (rural/urban); ${ }^{6} p$-values for the adjusted OR; $p$-values $<0.00061$ are highlighted in bold as significant according to Bonferroni correction for multiple comparisons;

${ }^{7}$ chronic obstructive pulmonary disease; ${ }^{8}$ acute myocardial infarction.

\section{Discussion}

In this large-scale population-based study, we used real-world data to analyze AD prevalence in 216,291 pediatric users of the Spanish public health system. We exhaustively characterized the comorbidities of all 33,591 boys and girls with AD in the EpiChron Cohort, with a focus on the most prevalent chronic diseases and on those that were associated the most to AD regardless of their prevalence.

The prevalence of AD obtained in our population (15.5\%) was similar to that found in other industrialized countries such as Sweden and Japan (16\%) [7]. The soundest study to date in Spain on $\mathrm{AD}$ prevalence in children reported slightly higher rates in 6-7-year-olds (28\%) than the ones shown by 3-9-year-olds in our population (22\%) [8]. However, while the former study used surveys completed by children's parents, we analyzed patients' clinical histories to extract professionally diagnosed cases of $\mathrm{AD}$, which could explain the variations in prevalence. Concurring with previous studies, and although prevalence differences between boys and girls were minimal in our population, girls were more affected by $\mathrm{AD}$ than boys $[22,23]$.

Surprisingly, AD prevalence was similar in native (Spanish) and Asian or sub-Saharan African children, who come from areas of traditionally low prevalence [7]. Our results seem to support the theory that environmental factors related to industrialization could increase the risk of AD in people who migrate to industrialized countries [24]. However, we had no data on the immigration status of children or the age at which they migrated to Spain. This hypothesis also concurs with the well-known "hygiene theory" and with the slightly higher AD prevalence rates found in children from urban areas 
compared to rural environments $[23,25]$. Similarly, our findings, which reflected higher AD prevalence rates in children belonging to higher socio-economic levels, were consistent with the results reported in seven-year-old children in Great Britain, who also showed differences in this regard [26]. One might expect that more readily available access to healthcare could account for the observed increase in the prevalence of $\mathrm{AD}$ in children from urban and/or less deprived settings. However, the public health system in Spain provides equal conditions of access to care regardless of the area and, therefore, we believe that said increase in prevalence would be due to other factors.

In order to offer a global characterization of the comorbidity profile present in these children, we followed a two-pronged approach; we described both the most common comorbidities irrespective of their association to $\mathrm{AD}$, as well as those with an increased risk of occurrence in children with a diagnosis of AD. The co-occurrence of these comorbidities could be a cause or a consequence of $\mathrm{AD}$ or share with it a common etiological mechanism. In line with previous studies, our investigation evidenced that children and adolescents with AD frequently suffer from multimorbidity ( $43 \%)$ and show an increased risk of developing a great number of diseases and symptoms of varying natures.

Prevalence rates of asthma, the most frequent AD comorbidity in our population, were lower $(13 \%)$ than those reported in 3-5-year-olds in two Italian studies (24\%) [27], and in 17-year-olds and under in the USA (25\%) [28]. Furthermore, we found that atopy-related comorbidity prevalence rates increased with age; asthma and allergic reactions debuted at ages 3-9, followed by allergic rhinitis in the group of 10-14-year-olds in a sequence of increasing prevalence rates for different atopic conditions known as the "atopic march" [29].

In consonance with several studies highlighting the importance of mental health disorders [30-33] and suicidal ideation [34] in children with $\mathrm{AD}$, psychosocial disorders were the second most prevalent chronic condition (7.9\%) in our study population. Although the determining factors of this association are diverse, previous studies suggest that sleep disturbances play a key role in mental health disorder development in children with AD [31,35-37], particularly in conditions such as ADHD [38]. ADHD, in fact, was also more frequent in our study in children with AD compared to those without. These and other psychiatric disorders can be present even in preschool children with atopic eczema, although in our sample they become a relevant comorbidity in 10-year-olds and above [39].

Regarding visual impairment, the third most frequent comorbidity in our study $(7.8 \%)$, Pols et al. [40] found an association between $\mathrm{AD}(\mathrm{OR}(95 \% \mathrm{CI}))$ and blepharitis/styes/chalazion (1.53 (1.29-1.81)), infectious conjunctivitis (1.53 (1.20-1.81)), and allergic conjunctivitis (1.99 (1.59-2.49)) after analyzing a nationwide primary care database of children in the Netherlands. However, since the exact prevalence of each of the diagnoses included in the visual impairment EDC was unavailable, we were unable to contrast their results with our own. Our results for irritable bowel syndrome (1.90 (1.56-2.31)) were consistent with other recently published studies on children and adults with AD [41,42].

Musculoskeletal comorbidities, such as congenital anomalies of limbs, hands, and feet, kyphoscoliosis, degenerative joint disease, and lower back pain were more frequent in AD pediatric patients, who were shown to share common risk factors for injuries, including the aforementioned sleep disturbances, distractions due to chronic itch, the sedative effects of antihistamine drugs, and psychological comorbidities [43]. Additionally, low vitamin D blood levels in AD patients might also contribute to the skeletal burden [44]. Pols et al. found a higher prevalence of foot/toe-related symptoms (1.39 (1.20-1.60)) and acquired deformities of the limbs (1.39 (1.20-1.60)) in their study [40]; however, the association between $\mathrm{AD}$ and congenital limb anomalies or kyphoscoliosis was not established.

Otological conditions, both acute and chronic, were also more prevalent in patients with AD. Otitis media was present in $13 \%$ of patients in our sample and was especially significant in 0-9-year-olds, showing higher rates than those found by Silverberg et al. in the United States of America (USA) (5\%) [45]. A recent systematic review also found a higher risk of infectious otitis in patients with AD [45], whereas deafness and hearing loss, which had an adjusted OR of 1.52 (1.39-1.66) in our sample, had not been previously associated with AD. 
The prevalence of autoimmune diseases is reportedly higher in children with AD [46]. Alopecia areata frequently shows the strongest association [46,47], which would be consistent with the higher prevalence of hair and hair follicles diseases found in our sample. Psoriasis was also more prevalent in our population with $\mathrm{AD}$, as previously described by Pols et al. [40]. The association of central obesity with AD was already reported, especially in women [16]. A recent study published by our group on 14-year-olds and below concluded that the prevalence of overweight, obesity, and dyslipidemia was greater in those with AD [48], which concurs with our present findings.

We have to highlight the existence of some comorbidities of varying nature that, although not significant after applying the Bonferroni correction for multiple comparisons, deserve special attention since they could be potentially associated with AD, as they presented some of the highest ORs of our study. This is the case of chronic cystic disease of the breast (2.61 (1.13-6.06)) that, despite its very low prevalence $(0.02 \%)$, was the condition $\mathrm{AD}$ was associated to the most, and which had not been previously reported in other studies that we know of. Congenital heart diseases and cardiac valve disorders also presented high ORs and had not been described in the existing literature either. As certain authors already suggested, AD could potentially constitute a risk factor for the development of cardiovascular diseases during adulthood $[15,49]$. Inflammatory bowel disease, although infrequent, also showed one of the highest adjusted ORs (1.86 (1.01-3.45)). Previous studies support the association of both diseases in children $[46,50]$ and, in fact, inflammatory bowel disease shares 39 genetic risk loci with $\mathrm{AD}$, also suggesting a genetic cause [51].

Our results could help better understand the comorbidity of $\mathrm{AD}$ and improve its overall management and treatment, providing patients with holistic and person-centered care. Nonetheless, statistical associations do not necessarily translate into the existence of causal relationships, and these findings should be interpreted with caution. Although many common underlying factors are well documented in the existing literature, some of the associations found in our study were not previously considered and could set the basis for future studies aimed at identifying common pathophysiological pathways connecting AD to other conditions of varying nature.

The main strength of our work lies in its large-scale, population-based characteristics, as it included almost every child with an AD diagnosis belonging to the reference population area. Moreover, AD comorbidity was exhaustively analyzed through the study of every chronic disease diagnosis in both primary and hospital care, not only the most prevalent or relevant ones. The use of EHRs guaranteed the reliability of data, which underwent continuous quality control revisions to ensure its accuracy. The main limitation of our study was its cross-sectional nature, which made determining the chronological order of appearance of diseases impossible, thus hindering the identification of cause-effect relationships between AD and its comorbidities. Furthermore, EHRs were not specifically designed for the purposes of this research, thus leading to potential over- or under-reporting of certain diseases. Moreover, some variables of interest for the study, such as lifestyle habits (e.g., lack of exercise, unhealthy eating), environmental information (e.g., air pollution, weather conditions), or specific data on immigration status of children were not available in the cohort.

\section{Conclusions}

The present study evidences that $\mathrm{AD}$ is prevalent in children and that it is systematically associated with some relevant non-dermatological conditions of neuropsychiatric, visual, cardio-metabolic, and autoimmune nature, among others. Our results highlight the importance of monitoring the array of comorbidities surrounding AD during childhood, which could help improve their clinical management and plan interventions aimed at preventing their development. Future longitudinal studies are encouraged to shed light on the underlying pathophysiological mechanisms involved in these associations.

Supplementary Materials: The following are available online at http://www.mdpi.com/2077-0383/9/6/1632/s1: Table S1. Prevalence of the 10 most frequent diseases (both acute or chronic) in children with atopic dermatitis, according to age and sex. 
Author Contributions: Conceptualization, Y.G. and A.P.-T.; methodology, A.G.-M. and B.P.-P.; formal analysis, B.P.-P. and J.B.P.-G.; data curation, B.P.-P.; writing-original draft preparation, Y.G. and J.B.P.-G.; writing-review and editing, A.G.-M., K.B.-B., and A.P.-T.; funding acquisition, A.P.-T. and Y.G. All authors read and agreed to the published version of the manuscript.

Funding: This work was supported by Gobierno de Aragón (B01_20R and B18_17D) and the European Regional Development Fund "Construyendo Europa desde Aragón".

Acknowledgments: The authors sincerely thank Eva Giménez Labrador for her statistical support.

Conflicts of Interest: The authors declare no conflicts of interest. The funders had no role in the design of the study; in the collection, analyses, or interpretation of data; in the writing of the manuscript, or in the decision to publish the results.

\section{References}

1. Lewis-Jones, S. Quality of life and childhood atopic dermatitis: The misery of living with childhood eczema. Int. J. Clin. Pract. 2006, 60, 984-992. [CrossRef]

2. Sánchez-Pérez, J.; Daudén-Tello, E.; Mora, A.M.; Lara Surinyac, N. Impacto de la calidad de vida relacionada con la salud en población pediátrica y adulta española con dermatitis atópica. Estudio PSEDA. Actas Dermosifiliogr. 2013, 104, 44-52. [CrossRef]

3. Kemp, A.S. Cost of illness of atopic dermatitis in children: A societal perspective. Pharmacoeconomics 2003, 21, 105-113. [CrossRef]

4. Asher, M.I.; Montefort, S.; Björkstén, B.; Lai, C.K.; Strachan, D.P.; Weiland, S.K.; Williams, H. Worldwide time trends in the prevalence of symptoms of asthma, allergic rhinoconjunctivitis, and eczema in childhood: ISAAC Phases One and Three repeat multicountry cross-sectional surveys. Lancet 2006, 368, 733-743. [CrossRef]

5. Nutten, S. Atopic dermatitis: Global epidemiology and risk factors. Ann. Nutr. Metab. 2015, 66, 8-16. [CrossRef]

6. Odhiambo, J.A.; Williams, H.C.; Clayton, T.O.; Robertson, C.F.; Asher, M.I.; ISAAC Phase Three Study Group. Global variations in prevalence of eczema symptoms in children from ISAAC Phase Three. J. Allergy Clin. Immunol. 2009, 124, 1251.e23-1258.e23. [CrossRef]

7. Bagazgoitia, L.; Gutiérrez, M.; García Blesa, C.; Hernández Martín, A.; Torrelo, A. Epidemiologic, pathogenic, clinical and diagnostic aspects of atopic dermatitis. Is it possible the prevention? Rev. Pediatr. Aten. Primaria 2009, 11, 31-47. [CrossRef]

8. Suárez-Varela, M.M.; García-Marcos Alvarez, L.; Kogan, M.D.; González, A.L.; Gimeno, A.M.; Ontoso, I.A.; Díaz, C.G.; Pena, A.A.; Aurrecoechea, B.D.; Monge, R.M.B.; et al. Climate and prevalence of atopic eczema in 6- to 7-year-old school children in Spain. ISAAC PhASE III. Int. J. Biometeorol. 2008, 52, 833-840. [CrossRef]

9. Rivero Gairaud, J.I. Dermatitis atópica. Rev. Médica Costa Rica y Cent. 2016, 83, 711-716.

10. Rojas, A.R.; Quezada, L.A. Relación entre dermatitis atópica y alergia alimentaria. Rev. Chil. Pediatría 2013, 84, 438-450. [CrossRef]

11. Pérez-Cotapos, S.M.L.; Zegpi, T.M.S.; Santa María, M.L.S. De Dermatitis atópica. Rev. Médica Clínica Las Condes 2011, 22, 197-203. [CrossRef]

12. Sociedad Mexicana de Alergia e Inmunología Pediátrica, I.L.; Mendoza Hernández, D.; López Pérez, G.T.; Carmona Barrón, M. Dermatitis atópica y comorbilidades en el paciente pediátrico. Alergia Asma e Inmunol. Pediátricas 2018, 27, 71-78.

13. Chen, J.J.; Applebaum, D.S.; Sun, G.S.; Pflugfelder, S.C. Atopic keratoconjunctivitis: A review. J. Am. Acad. Dermatol. 2014, 70, 569-575. [CrossRef]

14. Shalom, G.; Dreiher, J.; Kridin, K.; Horev, A.; Khoury, R.; Battat, E.; Freud, T.; Comaneshter, D.; Cohen, A.D. Atopic dermatitis and the metabolic syndrome: A cross-sectional study of 116816 patients. J. Eur. Acad. Dermatol. Venereol. 2019, 33, 1762-1767. [CrossRef]

15. Paller, A.; Jaworski, J.C.; Simpson, E.L.; Boguniewicz, M.; Russell, J.J.; Block, J.K.; Tofte, S.; Dunn, J.D.; Feldman, S.R.; Clark, A.R.; et al. Major Comorbidities of Atopic Dermatitis: Beyond Allergic Disorders. Am. J. Clin. Dermatol. 2018, 19, 821-838. [CrossRef]

16. Ali, Z.; Suppli Ulrik, C.; Agner, T.; Thomsen, S.F. Is atopic dermatitis associated with obesity? A systematic review of observational studies. J. Eur. Acad. Dermatol. Venereol. 2018, 32, 1246-1255. [CrossRef] 
17. Prados-Torres, A.; Poblador-Plou, B.; Gimeno-Miguel, A.; Calderón-Larrañaga, A.; Poncel-Falcó, A.; Gimeno-Feliú, L.A.; González-Rubio, F.; Laguna-Berna, C.; Marta-Moreno, J.; Clerencia-Sierra, M.; et al. Cohort Profile: The Epidemiology of Chronic Diseases and Multimorbidity. The EpiChron Cohort Study. Int. J. Epidemiol. 2018, 47, 382-384f. [CrossRef]

18. The Johns Hopkins ACGßSystem. Available online: https://www.hopkinsacg.org/ (accessed on 8 August 2017).

19. Eichenfield, L.F.; Tom, W.L.; Chamlin, S.L.; Feldman, S.R.; Hanifin, J.M.; Simpson, E.L.; Berger, T.G.; Bergman, J.N.; Cohen, D.E.; Cooper, K.D.; et al. Guidelines of care for the management of atopic dermatitis: Section 1. Diagnosis and assessment of atopic dermatitis Work Group. J. Am. Acad. Dermatol. 2014, 70, 338-351. [CrossRef]

20. Compés Dea, M.L.; Olivan Bellido, E.; Feja Solana, C.; Aguilar Palacio, I.; García-Carpintero Romero del Hombrebueno, G.; Adiego Sancho, B. Construction of a deprivation index by Basic Healthcare Area in Aragon using Population and Housing Census 2011. Rev. Esp. Salud Publica 2018, 92, 1-17.

21. Salisbury, C.; Johnson, L.; Purdy, S.; Valderas, J.M.; Montgomery, A.A. Epidemiology and impact of multimorbidity in primary care: A retrospective cohort study. Br. J. Gen. Pract. 2011, 61, e12-e21. [CrossRef]

22. Martorell Aragonés, A.; Félix Toledo, R.; Martorell Calatayud, A.; Cerdá Mir, J.C. Epidemiologic, clinical and socioeconomic factors of atopic dermatitis in Spain: Alergológica-2005. J. Investig. Allergol. Clin. Immunol. 2009, 19 (Suppl. 2), 27-33.

23. Pomavilla Duy, M.C.; Torres Gutama, L.C. Prevalencia de Dermatitis Atópica y el Estudio de Sus Comorbilidades en Pacientes de 3 Meses a 16 Años de Consulta Externa de Dermatología del Hospital Vicente Corral Moscoso; Universidad de Cuenca: Cuenca, Spain, 2015.

24. Stefanovic, N.; Flohr, C.; Irvine, A.D. The exposome in atopic dermatitis. Allergy 2019. [CrossRef]

25. Mei-Yen Yong, A.; Tay, Y.K. Atopic Dermatitis: Racial and Ethnic Differences. Dermatol. Clin. 2017, 35, 395-402. [CrossRef]

26. Mercer, M.J.; Joubert, G.; Ehrlich, R.I.; Nelson, H.; Poyser, M.A.; Puterman, A.; Weinberg, E.G. Socioeconomic status and prevalence of allergic rhinitis and atopic eczema symptoms in young adolescents. Pediatr. Allergy Immunol. 2004, 15, 234-241. [CrossRef]

27. Peroni, D.G.; Piacentini, G.L.; Bodini, A.; Rigotti, E.; Pigozzi, R.; Boner, A.L. Prevalence and risk factors for atopic dermatitis in preschool children. Br. J. Dermatol. 2008, 158, 539-543. [CrossRef]

28. Silverberg, J.I.; Simpson, E.L. Association between severe eczema in children and multiple comorbid conditions and increased healthcare utilization. Pediatr. Allergy Immunol. 2013, 24, 476-486. [CrossRef]

29. Spergel, J.M.; Paller, A.S. Atopic dermatitis and the atopic march. J. Allergy Clin. Immunol. 2003, 112, S118-S127. [CrossRef]

30. Garg, N.; Silverberg, J.I. Association between childhood allergic disease, psychological comorbidity, and injury requiring medical attention. Ann. Allergy Asthma Immunol. 2014, 112, 525-532. [CrossRef]

31. Yaghmaie, P.; Koudelka, C.W.; Simpson, E.L. Mental health comorbidity in patients with atopic dermatitis. J. Allergy Clin. Immunol. 2013, 131, 428-433. [CrossRef]

32. Augustin, M.; Radtke, M.A.; Glaeske, G.; Reich, K.; Christophers, E.; Schaefer, I.; Jacobi, A. Epidemiology and Comorbidity in Children with Psoriasis and Atopic Eczema. Dermatology 2015, 231, 35-40. [CrossRef]

33. Schmitt, J.; Apfelbacher, C.; Chen, C.-M.; Romanos, M.; Sausenthaler, S.; Koletzko, S.; Bauer, C.-P.; Hoffmann, U.; Krämer, U.; Berdel, D.; et al. Infant-onset eczema in relation to mental health problems at age 10 years: Results from a prospective birth cohort study (German Infant Nutrition Intervention plus). J. Allergy Clin. Immunol. 2010, 125, 404-410. [CrossRef] [PubMed]

34. Rønnstad, A.T.M.; Halling-Overgaard, A.S.; Hamann, C.R.; Skov, L.; Egeberg, A.; Thyssen, J.P. Association of atopic dermatitis with depression, anxiety, and suicidal ideation in children and adults: A systematic review and meta-analysis. J. Am. Acad. Dermatol. 2018, 79, 448.e30-456.e30. [CrossRef] [PubMed]

35. Reuveni, H.; Chapnick, G.; Tal, A.; Tarasiuk, A. Sleep fragmentation in children with atopic dermatitis. Arch. Pediatr. Adolesc. Med. 1999, 153, 249-253. [CrossRef] [PubMed]

36. Yokomaku, A.; Misao, K.; Omoto, F.; Yamagishi, R.; Tanaka, K.; Takada, K.; Kohyama, J. A study of the association between sleep habits and problematic behaviors in preschool children. Chronobiol. Int. 2008, 25, 549-564. [CrossRef]

37. Hiscock, H.; Canterford, L.; Ukoumunne, O.C.; Wake, M. Adverse associations of sleep problems in Australian preschoolers: National population study. Pediatrics 2007, 119, 86-93. [CrossRef] 
38. Camfferman, D.; Kennedy, J.D.; Gold, M.; Martin, A.J.; Winwood, P.; Lushington, K. Eczema, sleep, and behavior in children. J. Clin. Sleep Med. 2010, 6, 581-588. [CrossRef]

39. Catal, F.; Topal, E.; Soylu, N.; Ozel Ozcan, O.; Celiksoy, M.H.; Babayiğit, A.; Karakoç, H.T.E.; Erge, D.; Sancak, R. Psychiatric disorders and symptoms severity in preschool children with atopic eczema. Allergol. Immunopathol. 2016, 44, 120-124. [CrossRef]

40. Pols, D.H.J.; Bohnen, A.M.; Nielen, M.M.J.; Korevaar, J.C.; Bindels, P.J.E. Risks for comorbidity in children with atopic disorders: An observational study in Dutch general practices. BMJ Open 2017, 7, e018091. [CrossRef]

41. Tsai, J.-D.; Wang, I.-C.; Shen, T.-C.; Lin, C.-L.; Wei, C.-C. A 8-year population-based cohort study of irritable bowel syndrome in childhood with history of atopic dermatitis. J. Investig. Med. 2018, 66, 755-761. [CrossRef]

42. Kaya İslamoğlu, Z.G.; Unal, M.; Küçük, A. Atopic Dermatitis in Adults and Irritable Bowel Syndrome: A Cross-sectional Study. Indian J. Dermatol. 2019, 64, 355-359. [CrossRef]

43. Silverberg, J.I. Selected comorbidities of atopic dermatitis: Atopy, neuropsychiatric, and musculoskeletal disorders. Clin. Dermatol. 2017, 35, 360-366. [CrossRef]

44. Baïz, N.; Dargent-Molina, P.; Wark, J.D.; Souberbielle, J.C.; Annesi-Maesano, I. Cord serum 25-hydroxyvitamin $\mathrm{D}$ and risk of early childhood transient wheezing and atopic dermatitis. J. Allergy Clin. Immunol. 2014, 133, 147-153. [CrossRef]

45. Silverberg, J.I.; Silverberg, N.B. Childhood atopic dermatitis and warts are associated with increased risk of infection: A US population-based study. J. Allergy Clin. Immunol. 2014, 133, 1041-1047. [CrossRef]

46. Narla, S.; Silverberg, J.I. Association between atopic dermatitis and autoimmune disorders in US adults and children: A cross-sectional study. J. Am. Acad. Dermatol. 2019, 80, 382-389. [CrossRef]

47. Wohlmuth-Wieser, I.; Osei, J.S.; Norris, D.; Price, V.; Hordinsky, M.K.; Christiano, A.; Duvic, M. Childhood alopecia areata-Data from the National Alopecia Areata Registry. Pediatr. Dermatol. 2018, 35, 164-169. [CrossRef]

48. Agón-Banzo, P.J.; Sanmartin, R.; García-Malinis, A.J.; Hernández-Martín, Á.; Puzo, J.; Doste, D.; Pardos, C.; Gilaberte, Y. Body mass index and serum lipid profile: Association with atopic dermatitis in a paediatric population. Australas. J. Dermatol. 2019. [CrossRef]

49. Silverberg, J.I.; Gelfand, J.M.; Margolis, D.J.; Boguniewicz, M.; Fonacier, L.; Grayson, M.H.; Simpson, E.L.; Ong, P.Y.; Chiesa Fuxench, Z.C. Association of atopic dermatitis with allergic, autoimmune, and cardiovascular comorbidities in US adults. Ann. Allergy Asthma Immunol. 2018, 121, 604.e3-612.e3. [CrossRef]

50. Kappelman, M.D.; Galanko, J.A.; Porter, C.Q.; Sandler, R.S. Association of paediatric inflammatory bowel disease with other immune-mediated diseases. Arch. Dis. Child. 2011, 96, 1042-1046. [CrossRef]

51. Paternoster, L.; Standl, M.; Waage, J.; Baurecht, H.; Hotze, M.; Strachan, D.P.; Curtin, J.A.; Bønnelykke, K.; Tian, C.; Takahashi, A.; et al. Multi-ancestry genome-wide association study of 21,000 cases and 95,000 controls identifies new risk loci for atopic dermatitis. Nat. Genet. 2015, 47, 1449-1456. 